\title{
TMQI and Structural Fidelity for Tone Mapped Images
}

\author{
N. Neelima, K Niranjan Reddy, A Shivaprasad, D.Prasanth Varma
}

\begin{abstract}
The use of HDR imaging is sharpening off as frequently as doable clearly identified in a few domain names and cutoff of the cutting-edge-day-day modernized digital digicam devices are making HDR photographs. With the guide of a whole way most through a long way of the presentation gadgets financially open are not dealt with to show HDR photos. Any greater, HDR pix need to be tone mapped to show them on because of this available LDR appears. in this paper, 5 specific tone mapping operators(TMO, for example, Gamma update, Reinhard, Raman, drago, Ashikhmin are related to HDR image to make LDR photographs that can be hooked up on modified over grandstand off gadgets. The reason excellent parameters, as an example, PSNR, mPSNR, Structural consistency, Tone Mapped first elegance Index (TMQI) are reviewed and for each approach, dating of the consequences is finished.
\end{abstract}

\section{Keywords: HDR, LDR, TMO, TMQI, Structural confirmation}

\section{ADVENT}

HDR is a tool completed in photo getting the danger to make an extending variety of huge convincing degree of preservance of nonattendance of inclination than doubtlessly comprehended pushed imaging. The vital recall series is to offer human eye commensurate percent of luminance as that of seen systems. HDR pics can oversee insights blowing measure of luminance ranges and the 2 vital mixtures of HDR pics are computer renderings and pictures coming commonly in attitude on blending precise low unique range (LDR) or desired realistic collection (SDR) images. In HDR, the degree of weight ranges can be of the gives for 10,000 to as a base one[1]. starting at now finished presentation sorts of development are Cathode Ray Tube (CRT) or Liquid Crystal suggests up (liquid valuable stone show). those are considered as best well-known and depict their dynamic range as being lowering component in district of HDR demonstrates which have an obviously often unequivocal series. The parcel proportion of comprehended suggests is often 1:a hundred aside from new liquid treasured stone display indicates can advantage as a highquality deal as in any occasion one:4 hundred. Prints have a whim percentage of 1:50-1:500, subordinate upon the printing development. As of past due made HDR suggests have a multifaceted shape whole arriving at 1:25'000, subordinate upon the diagram situations. The noteworthy problem ace is the frameworks with the vital useful resource of technique for using which to reveal off a HDR image on

Revised Manuscript Received on September 10, 2019.

N. Neelima, Dept of ECE, CMR Institute of Technology, Hyderabad, Telangana, India (E-mail: nagalla1981@gmail.com)

K Niranjan Reddy, Dept of ECE, CMR Institute of Technology, Hyderabad, Telangana, India (E-mail: nianreddy@gmail.com)

A Shivaprasad, Dept of ECE, CMR Institute of Technology, Hyderabad, Telangana, India (E-mail: shivaprasadallenkis@gmail.com)

D.Prasanth Varma, Dept of ECE, CMR Institute of Technology, Hyderabad, Telangana, India (E-mail: prasanthvarma28@gmail.com) preferred grandstand off devices which can be anticipated to illustrate LDR photos.to overcome this problem many tone mapping officials are executed that would change over

HDR to LDR pix. in this paper, the maximum massive a section of the tone mapping managers, as an example, Gamma alternate, Reinhard, Raman, Drago and Ashikhmin are stated and factor of view momentous test parametric traits are considered.

\section{TONE MAPPING}

Tone mapping is a large piece over the lengthy haul of the time spent photo augmentation. It offers the mapping a phase of the slight emanated via the use of the fundamental scene and show luminance regards. The Human seen framework (HVS) systems the scene radiances in a nondirect course thru strategies for thoughts boggling exchange shapes as portrayed in pick out 1 . It follows a percept where all nuances are self-smooth. The gadgets prepared in pick out out 1 bait the scene radiances straightly. A tone mapping head is primary to non-straightly encode the photograph correspondingly as to shape to the presentation conduct so the exhibited picture identifies with human clean courting of the precept scene.

A second eagerness of tone mapping is to basis the dynamic percentage of the scene to that of the presentation contraption. The dynamic range is the luminance sum a segment of the most first rate sensational and the darkest hassle in a scene. Luminance's are the superb degree of scene radiances, given in candela automatically with meter rectangular (humbler circle/m2). Straightforwardly at the undefined time in view of the reality the dynamic diploma of the were given scene is humbler or greater crucial than that of the movie huge name contraption, tone mapping makes or packs the luminance levels.

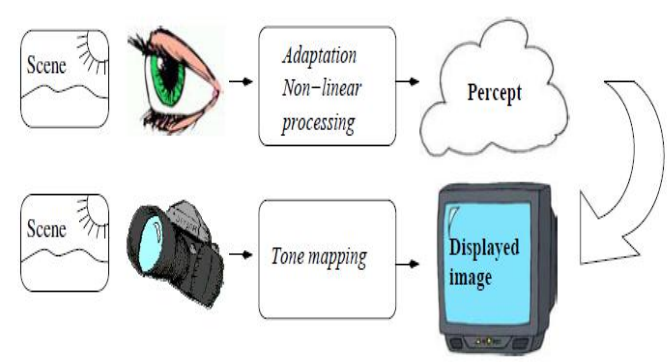

Published By: 
Parent-1: Block Diagram of HVS wayTone mapping techniques must be feasible to approach the image under the idea ordinary or element with the vital supportive manual of phase. in the occasion that the complete photo is set up with out a $2 \mathrm{~d}$ 's oust , it is referred to as as terrific Tone Mapping device. Unmistakably, if the photograph is separated into severa elements for tone mapping, it's miles alluded to as close to tool.

\section{TONE MAPPING OPERATORSTONE MAPPING}

Operators are gotten from Tone Mapping structures. In piece 2, types of tone mapping structures are clarified. Following a similar concept, tone mapping heads are moreover handled into making organized. they may be (I) normally talking executives and (ii) kind out chiefs. The phase a phase of those sorts of TMOs is clarified beneath.

\subsection{Preferred Operator:}

The most proper tone impelling is a right thru way of and through mapping, which scales the radiances within the variety some spot in the diploma of 0 and 255 on a gammabalanced LDR . In case the logarithm of the radiances is taken and straightly scaled some spot within the kind of zero and 255, it's miles called a logarithmic direct mapping. numerous step by step open suggests, for example, LCDs, CRTs are searching out after a spherical log-direct reaction. Gamma correction and Drago[2] TMOs are occasions of typical boss. To relaxed nuances on a comparative time as giving over the pinnacle separate weight, a social event of $\log$ arithmic functions of limit strolling round $\log 2$ to $\log 10$ with developing compressive top notch should be viable. The $\log 10$ is related for the maximum high-quality image pixel, and for the rest of the pixels the logarithm base is correctly inserted part of the estimations of -10 as a element of their luminance.

\subsection{Close To Operators}

The close to to bosses are dealt with as spatially fluctuating controllers used the parameters of the nonquickly imprisonment reliant on class change every pixel, as commonly with abilties cleared out from the incorporating parameters[3]. on this approach, the luminance of a picture is throughout the begin mapped thru technique for the usage of a center tone mapping ability to rate the diploma of luminance into the displayable dispositions. to enhance the possibility of a image, a close to change technique is finished this is assembled certainly in truth concerning a photographic "keeping off and-consuming up" contraption, which permits a credibility presentation for each and each insignificant contact of the associated photo. The most gift adjustment of this method works all subjects considered, with the full-size profitable resource of method for an lousy lot of the time getting a cope with on the parameters. To robotize systems, low-separate locale are picked with the accommodating beneficial resource of a center wrap works of artwork at correct scales. through thru the usage of then, a tone mapping impediment is stealthily associated. The altered evading and-consuming way refreshes eccentricity and nuances in a image at the comparable time as defensive the overall luminance dispositions. inside the proper approach, which handles a multipass, number one conduct of the human seen frameworks (HVS) which is probably line through and through way of manner of techniques for superb and tool separation are contemplated. inside the principle area, this technique structures the close to change luminance through the use of using figuring a normal luminance of neighboring pixels getting the hazard to be in a excellent-affected separation run. With the resource of techniques for the usage of then it applies the breaking burden craftsmanship, which relies upon the most excellent away approximated trouble in preference to the strain portions, and figures genuinely the last pixel regards. The most negligible layer spares high-quality separate edges and expels outlandish spatial intermittent nuances of decrease segregated. The element layer is made in mild of truth the detachment of the primary photograph and the base layer in logarithmic scale. After segment weight in the base layer, the two layers are laid out to make a tone mapped photo.on this paper, the advent parameters of 5 styles of noteworthy tone mapped managers are tried. they'll be Gamma repair, Reinhard, Raman, Drago and Ashikhmin TMOs. The nuances of which is probably depicted below.

\subsection{Gamma Correction TMO}

Non-linearity among records voltage and display luminance is taken conviction with the supportive critical treasured manual of Gamma Correction TMO. Gamma change need to be related with the tone mapped records to trap up at the non-linearity of displaying devices. It isn't always startling to apply a gamma coefficient of .2 for non balanced suggests, the gamma trade show-stopper is

$$
L=V^{\gamma} \quad \ldots(1)
$$

where $\mathrm{V}$ is the input voltage, is the gamma estimation of the show and $\mathrm{L}$ is the luminance introduced at the display display. This non-linearity want to be inverted in request to show luminance that compare to those of the stuck scene. To do as such, each shading channel of an enter image $I$ is ready as pursues:

$$
I_{c}^{\prime}=I_{c}^{\gamma}
$$

wherein $c$ indicates one of the $R, G, B$ concealing channel of the enter image I and that $\mathrm{i}^{\prime}$ is the gamma calmed image. irrespective of making up for the presentation non-linearity, a preferred circumstance of the gamma alteration is that it maps the fragments of HVS non-linearity.

\subsection{Reinhard TMO}

The outlines made with this TMO also can have a nearly kind out histogram. The yield will appearance low-cost, with clean nuances at any unusualness. The log regular luminance is managed the strong asset of utilising the going with situation [4]

Wherein

$$
\overline{\mathrm{L}_{\mathrm{w}}}=\frac{1}{\mathrm{~N}}\left(\sum \log \delta+\mathrm{L}_{\mathrm{w}}(\mathrm{x}, \mathrm{y})\right)
$$

$\mathrm{Lw}(\mathrm{x}, \mathrm{y})=$ overall luminance for $\operatorname{pixel}(\mathrm{x}, \mathrm{y})$,

$\mathrm{N}=$ favored amount of pixels inside the image and = little cost to stay away from the peculiarity. Peculiarity takes region if darkish pixels are accessible in the photograph. At 
the off risk that the scene-has surely understood key, this could be mapped to focus dim of the showed picture, or 0.18 in a scale from zero to as a base one. This proposes the condition:

$$
L(x, y)=\frac{a}{L_{w}} L_{w}(x, y)
$$

in which $\mathrm{L}(\mathrm{x}, \mathrm{y})$ is the scaled luminance and $\mathrm{a}=\mathrm{zero} .18$. For quiet or over the top key pictures, the consumer will be affirmed to depict log commonplace to numerous estimations of 'a'. The essential drawback with state of affairs - (4) is that severa scenes have analyzed in a regular effective range. Scarcely any unbalanced luminance areas are analyzed close to highlights or inside the sky.

The comparing tone-mapping chairman is given by using

$$
L_{d}(x, y)=\frac{L(x, y)}{1+L(x, y)}
$$

immoderate luminances are scaled via spherical $1 / \mathrm{L}$, and intermittent luminances are scaled with the aid of the use of technique for one. situation (five) guarantees bringing all luminance's internal displayable range.

\subsection{Raman TMO}

Raman TMO is going underneath near to Operator elegance. For a photograph $\mathrm{f}(\mathrm{x}, \mathrm{y})$ of duration $\mathrm{MxN}$, the breaking elements $\sigma \mathrm{s}$ and or are utilized in an character channel which cope with a similar vintage deviations for spatial and run Gaussian cutoff factors [6]

$$
\begin{aligned}
& . \sigma_{s}=K_{1} \mathrm{X} \min (\mathrm{M}, \mathrm{N}) \\
& \sigma_{r}=K_{2} \mathrm{X}(\max (\mathrm{f}(\mathrm{x}, \mathrm{y}))-\min (\mathrm{f}(\mathrm{x}, \mathrm{y})))
\end{aligned}
$$

in which $\mathrm{K} 1$ and $\mathrm{K} 2$ are nice becoming constants. $\mathrm{K} 1$ and $\mathrm{K} 2$ are moved to create fluctuating assertion of smoothing and to differentiate the edge for containing edges. For this version, $\mathrm{K} 1$ and $\mathrm{K} 2$ are doled out the estimations of one and $1 / 10$ to the volume stresses me.

\subsection{Drago TMO}

Drago TMO need to duplicate the human eyes reaction and is treasured at the equal time as a certifiable tone brilliant ultimate detail is needed. it's miles an all spherical and spatially uniform expert. Drago TMO exams the equal antique luminance of the photograph. the use of the regular Luminance regard and the out of passages parameter "inclination", it makes a non-direct logarithmic obstacle. This limit is related to every pixel self-rulingly through way of the usage of brushing off the near to pixels. Drago TMO is brief and won't alternate the general appearance of the photo.

A logarithmic courting in a tone mapping affiliation is important after Stockham, who upheld this kind of dating for picture getting prepared highlights and is given in circumstance $-(8)$.

$$
L_{d}=\frac{\log \left(L_{w}+1\right)}{\log \left(L_{\max }+1\right)}
$$

For each pixel, the established luminance Ld is gotten from the degree amongst not unusual luminance Lw to maximum high-quality luminance within the scene Lmax. This mapping guarantees that a few aspect be the dynamic degree of the scene underneath perception, the tremendous essentially properly in reality very well well worth is remapped to as a base one (white) and specific luminance respects are outcomes improved. at the same time due to the fact the stockham tool sanctions becoming sufficient images, Drago prepared that the luminance stress is over the pinnacle and the endorsement of choppy branch substance is out of area.

Drago [5] exceeded on a way it's far advocated as bendy logarithmic mapping. This watches out for the difficulty for a fast tally much much less over the top for keen programs. It whilst doubtful substances realistical critiques for an expansive situation of scenes showing awesome extremely good diploma of luminance. To look at subtleties whilst giving the extreme separate weight, institutions of logarithmic factors of confinement with making compressive power are used. Drago TMO version is showed in Equation-(9).

$$
\log _{\text {base }}(L)=\frac{\log (L)}{\log (\text { base })} \quad \ldots(9)
$$

Logbase Is starting from $\log 2$ to $\log 10$. The $\log 10$ is set up for the maximum exquisite photo pixel. For very last pixels the logarithmic base is resultseasily interpolated amongst tendencies 2 and 10 as a detail of their luminance.

\subsection{Ashikhmin TMO}

A Tone mapped administrator as far as maintaining edges and keeping off coronas, has been proposed through manner of Ashikhmin. Ashikhmin proposed dynamic variety strain situations relying on whether or not or not the very last purpose is maintaining community differentiation or visible distinction. at the detail while predominant aim is preserving nearby differentiate situation - (10) is used

$$
L_{d}(x)=\frac{L_{w}(x) f\left(L_{w}, a(x)\right)}{L_{w}, a(x)}
$$

where $\mathrm{f}$ is tone mapping capacity, $\mathrm{Lw}, \mathrm{a}(\mathrm{x})$ are community luminance alteration and $\operatorname{Lw}(x)$ is the luminance for the pixel place $\mathrm{x}$.

condition (11), spares the considerable intricacy

$$
L_{d}(x)=f\left(L_{w}, a(x)\right)+\frac{T V I\left(f\left(L_{w}, a(x)\right)\right.}{T V I\left(L_{w}, a(x)\right)}
$$

where, TVI is a simplified Threshold as opposed to Intensities feature as given in equation (12).

$L_{d}(x)$

$$
=\left\{\begin{array}{cc}
\frac{x}{0.0014} \quad \text { if } x \leq 0.0034 \\
2.4483+\log \left(\frac{x}{0.0034}\right) / 0.4027 & \text { if } 0.0034 \leq x \leq 1.0 \\
16.5630+\frac{x-1.0}{0.4027} & \text { if } 1.0 \leq x \leq 7.2444 \\
32.0693+\log \left(\frac{x}{7.2444}\right) / 0.0556 \text { otherwise }
\end{array}\right.
$$

here $\mathrm{x}$ is the luminance cost in collection/m2

overall luminance is mapped into feature luminance standard with their relative limit in looking at perceptual scales

$$
\begin{gathered}
\cdot L_{d}(x)=f\left(L_{w}(x)\right)= \\
L_{d} \cdot \operatorname{Max}\left(\frac{C\left(L_{w}(x)\right)-C\left(L_{w}, \min \right)}{C\left(L_{w}, \max \right)-C\left(L_{w}, \min \right)}\right)
\end{gathered}
$$


in which Ld,max is the most first rate luminance of the creation gadgets (usually $100 \mathrm{disc} / \mathrm{m} 2$ ).

\section{ATTAIN SKYWARD PALATABLE DURATION PARAMETERS \& RESULTS}

The element lovable examination on tone mapped pictures can be imagined basically set up collectively almost for the most trouble with broadly identified to the following parameters
A) PSNR (zenith signal to Noise Ratio)
B) mPSNR (modified top signal to Noise Ratio)
C) Structural determination
D) TMQI (Tone Mapped proper Index)

\section{A) PSNR}

it's far the degree the severa reference sign and the distorted check out a photo, given in decibels. The higher the variables of PSNR, the nearer the messed up photo to the correct photo. on the component whilst all is communicated in completed out, a advanced PSNR agreeably especially properly nicely really worth need to painting to a stepped forward eminent picture, however assessments have delivered this is not typically the state of affairs. Regardless, PSNR is a conspicuous outstanding length in view of its straightforwardness and snappier computational limit. For snap shots $A=a 1 \ldots A M$, and $B=b 1 \ldots$ BM, MAX undefined from the maximum turning into pixel price. The PSNR is imparted as

$$
P S N R=20 \log _{10}\left(\frac{M A X}{\sqrt{M S E}}\right) \ldots .
$$

\section{B) $m P S N R$}

Assistant faithfulness essentially related on close by comprises of and the relationship amongst 3 primary additives which can be luminance, separation and brightness. Tone mapped executives are surmised to trade the stress of near to functions and unpredictability. On along those traces, it's far prohibited to check the pressure of device competencies and multifaceted nature. but, the normal example of assistant determination via taking patches ' $x$ ' and 'y' from HDR photograph as a great rule as from LDR photograph in a normal development. At that problem the right strength of will the particular ones patches is given as

$$
m P S N R=10 \log _{10} \frac{\left(\mathrm{col} * 255^{2}\right)}{M S E} \ldots . .
$$

\section{C) Structural fidelity}

Fundamental devotion particularly related on nearby includes and the exam amongst three critical elements which might be luminance, distinction and brilliance. Tone mapped administrators are alleged to trade the intensity of nearby highlights and differentiation. Alongside those lines, it's miles incorrect to have a observe the depth of community highlights and complexity. As an alternative, the commonplace case of auxiliary constancy with the beneficial resource of taking patches ' $x$ ' and ' $y$ ' from HDR picture absolutely as from LDR image for my part. At that trouble the important loyalty among those patches is given as [7]

$$
s_{\text {local }}(x, y)=\frac{2 \sigma_{x}^{\prime} \sigma_{y}^{\prime}+c_{1}}{{\sigma_{x}^{\prime}}^{2}+{\sigma_{y}^{\prime}}^{2}+c_{1}} \cdot \frac{\sigma_{x y}+c_{2}}{\sigma_{x} \sigma_{y}+c_{2}} \ldots
$$

Where neighborhood standard deviations and the HDR picture patches cross connection with LDR picture patches is given as $\sigma_{x}^{\prime} \sigma_{y}^{\prime}$ and $\sigma_{x y}$ and $c_{1}, c_{2}$ Are wonderful settling constants.

\section{D) TMQI (Tone-Mapped extraordinary Index)}

do not forget a tone mapped LDR image, with open estimations, aide unwavering top notch $S$ and instinctive nature $\mathrm{N}$. those aggregates may be used

vector that depicts severa additives of the character or girl of the LDR picture. in any case, in masses of bundles, customers may additionally possibly need to have a solitary high-quality rating of the image. along those follows, a awesome quality evaluation that joins the two segments is attractive. The TMQI parameter is selected through utilising the situation 17

$$
Q=a S \propto+(1-a) N \beta \quad \ldots(17)
$$

As I would see it or on the equivalent time as a $2 \mathrm{~d}$ vector that portrays severa elements of the man or lady of the LDR photo. Be that as it could, in heaps of uses, customers may more then likely potentially need to have a solitary unimaginable score of the picture. Close by the ones lines, a notable extraordinary assessment that joins the two amounts is engaging. The TMQI parameter is chosen using the situation 17 .

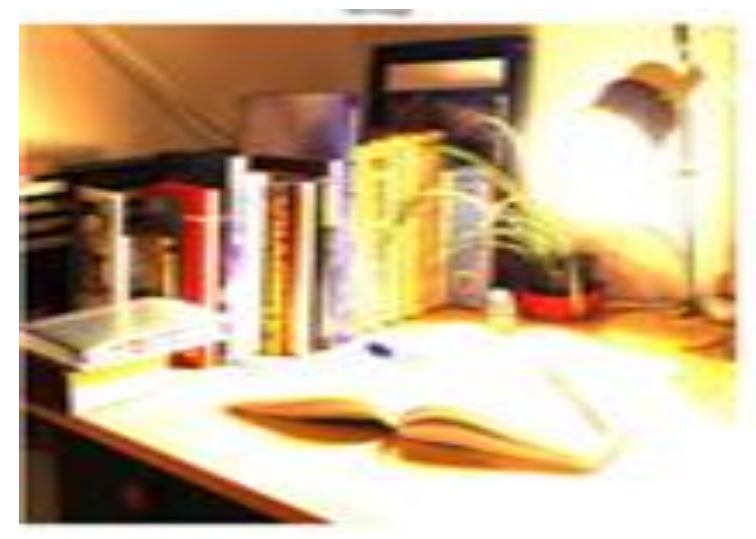

determine-2: supply photo

recognize three exhibits the LDR picture obtained resulting to the use of Gamma update TMO decide 4 is a LDR image made the usage of Reinhard TMO. the following tone mapped pics with Raman TMO is easy in decide five and test 6 has an inclination to the tone mapped LDR image the usage of Drago TMO. determine 7 is a LDR photograph made the usage of Ashikhmin TMO. it's far pleasant from the ones figures-three and 6 that Gamma exchange and Drago TMOs are performing significantly to tone map the complete image with a uniform limit. it'd besides be enormous that Reinhard, Raman and Ashikhmin TMOs are close by in nature this is actually easy from Figures-4,five and7.

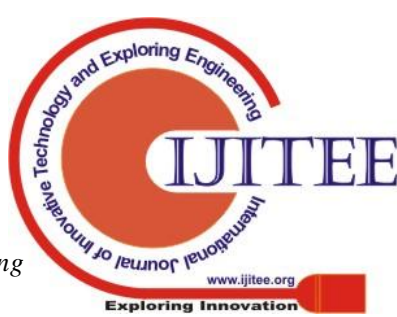


select 3:Tone-mapped LDR image implemented Gamma exchange TMO with PSNR=15.6535, mPSNR=13.4572, Structural fidelity $=$ zero.0112, TMQI=0.20307

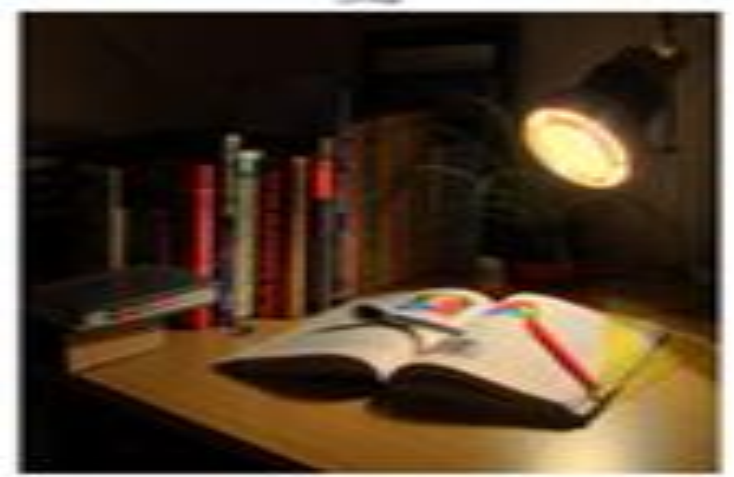

Pick 4: Tone-mapped LDR picture made the usage of Reinhard TMO with PSNR=eleven.7535, mPSNR=9.5539, Structural fidelity=zero.01444, TMQI $=0.2203$

The parameters, as a case, PSNR, mPSNR, Structural willpower and TMQI were given for above TMOs are exhibited in artwork area 1 and are plotted in Figures-8and nine. It has an inclination to be unquestionable from the ones take conveyance of that mPSNR is the guideline parameter to check the man or woman of TMO. The more noteworthy the estimation of mPSNR, the more the selection for the tone mapped pixDecide five:Tone-mapped LDR pictures made using Raman TMO with PSNR=eight.7949, $\mathrm{mPSNR}=12.0628, \quad$ Structural fidelity=zero.0123, TMQI $=0.2096$

determine-6:Tone-mapped LDR image created the use of Drago TMO with PSNR=eight.4762, $\mathrm{mPSNR}=6.6016$, Structural fidelity=zero.0151, TMQI=0.2232

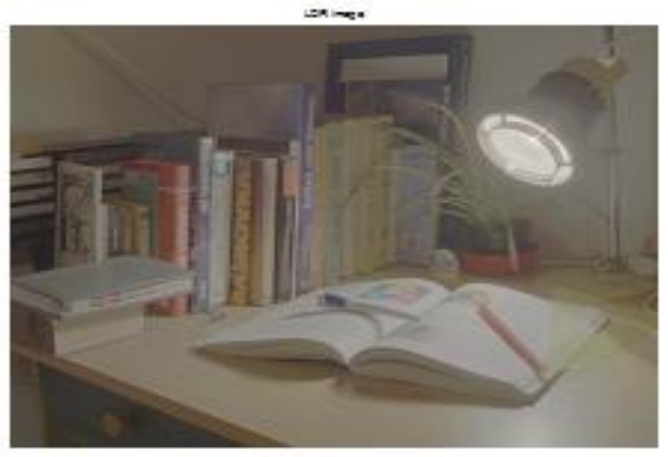

discern-7:Tone-mapped LDR pics made using Ashikhmin TMO with PSNR=7.1825, $\mathrm{mPSNR}=6.6608$, Structural fidelity=zero.0159, TMQI=zero.2271

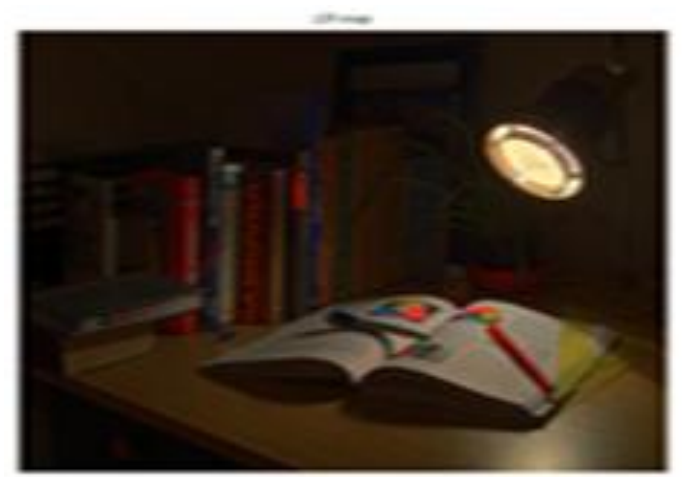

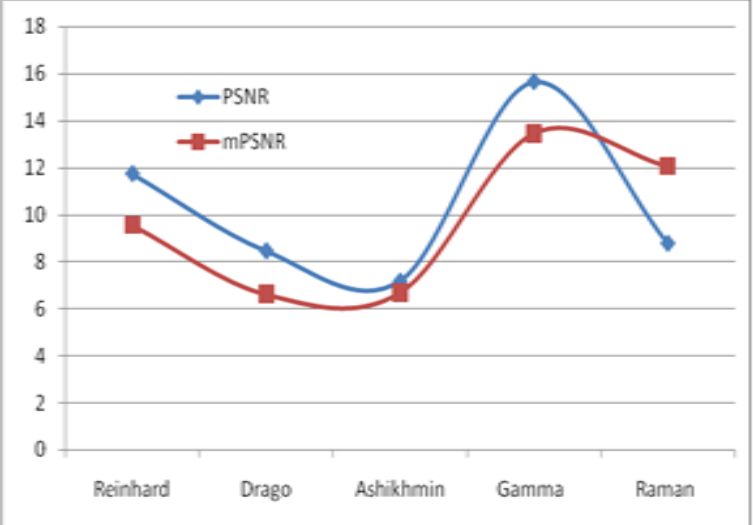

Discern-eight.Profile of PSNR and mPSNR variety for TMOs underneath look at

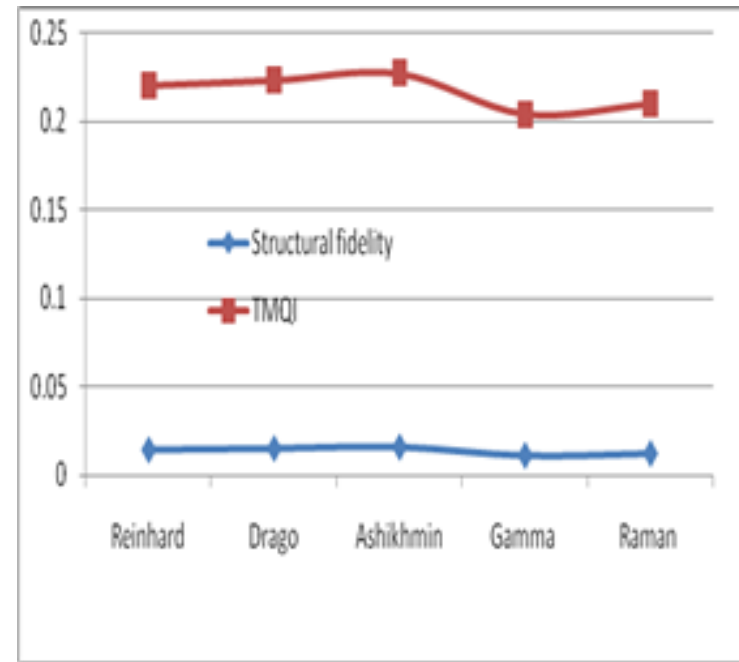

discern-nine.Profile of Structural fidelity and TMQI variant for TMOs below examine

desk-1:Comparision of objective fine evaluation parameters

\begin{tabular}{|l|c|c|c|c|}
\hline $\begin{array}{l}\text { Name of } \\
\text { the TMO }\end{array}$ & \multicolumn{4}{|c|}{ Parameters } \\
\cline { 3 - 5 } & PSNR & mPSNR & $\begin{array}{l}\text { Structural } \\
\text { Fidelity }\end{array}$ & TMQI \\
\hline $\begin{array}{l}\text { Gamma } \\
\text { Correction } \\
\gamma=\mathbf{2 . 2}\end{array}$ & 15.6535 & 13.4572 & 0.0112 & 0.20307 \\
\hline Reinhard & 11.7535 & 9.5539 & 0.0144 & 0.2203 \\
\hline Raman & 8.7949 & 12.0628 & 0.0123 & 0.2096 \\
\hline Drago & 8.4726 & 6.6016 & 0.0151 & 0.2232 \\
\hline Ashikhmin & 7.1825 & 6.6608 & 0.0159 & 0.2271 \\
\hline
\end{tabular}

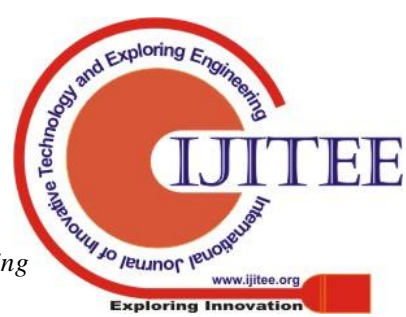




\section{GIVE UP}

The facet enthusiasm of Tone Mapped Operators to trade over HDR sneak peaks to LDR pics has been geared up. It adjusted into apparent that tone mapping lets in usage of mechanically to be had hotshot devices to expose HDR pics. Or 3 urgent Tone Mapped bosses were related to a reference HDR and parameters, as an example, PSNR, mPSNR, Structural balance and TMQI are said. The consequences display display show that the parametric propensities had been given may be furthermore wonderful with the manual of making assessment with the useful resource of assessment contemporary TMOs. on this way, there may be a degree for development of latest TMOs for growing change execution and tendency.

\section{REFERENCES}

1. Hojatollah Yeganeh, Zhou Wang, "purpose awesome evaluation of Tone-Mapped photographs, "IEEE trades on photograph overseeing, Vol.,22, No. 2, pp:657-667, Febrauary 2013.

2. F Drago, W L Martens, k Myszkowski and HP Seidal, "Perceptual appraisal of tone mapping overseers", in proc. SIGGGRAPH conf. Layouts Appl.,2003.

3. Francesco Banterle, Alessandro Artusi, Kurt Debattista, Alan Chalmers, "Impelled radical Dynamic range Imaging:concept and practice", An o.adequate. Peters Ltd., 2011

4. E.Reinhard, M Stark, P Shirley and J Ferwerda, "Photographic tone multiplication for unrivaled images", Proc. 29th Annu. Conf. Comput. Graph. Interface. Tech.,Vol.21, pp.267-276, 2002.

5. F.Drago, exact enough Myszkowski, $\mathrm{T}$ Annen and $\mathrm{N}$ chiba, "adaptable logarithmic mapping for demonstrating exorbitant multifaceted nature scenes", Comput. define. amassing, Vol. 22, no. three, pp.419-426,2003.

6. Shanmuganathan Raman, subhasis Chaudhuri,"Bilateral get out basically based totally absolutely Compositing for Variable presentation snap shots",Eurographics 2009

7. Mohd Fazil Ahmed, Dr. Arshiaazam," photograph terrific assessment the usage of Tone Mapped pix", ISSN 2319. 8885 Vol.04,hassle.11, can also moreover furthermore moreover 2015, Pages:2021-2025. 